\title{
A PARADOX IN THE APPROXIMATION OF DIRICHLET CONTROL PROBLEMS IN CURVED DOMAINS*
}

\author{
EDUARDO CASAS ${ }^{\dagger}$, ANDREAS GÜNTHER , AND MARIANO MATEOS $^{\S}$
}

\begin{abstract}
In this paper, we study the approximation of a Dirichlet control problem governed by an elliptic equation defined on a curved domain $\Omega$. To solve this problem numerically, it is usually necessary to approximate $\Omega$ by a (typically polygonal) new domain $\Omega_{h}$. The difference between the solutions of both infinite-dimensional control problems, one formulated in $\Omega$ and the second in $\Omega_{h}$, was studied in [E. Casas and J. Sokolowski, SIAM J. Control Optim., 48 (2010), pp. 3746-3780], where an error of order $O(h)$ was proved. In [K. Deckelnick, A. Günther, and M. Hinze, SIAM J. Control Optim., 48 (2009), pp. 2798-2819], the numerical approximation of the problem defined in $\Omega$ was considered. The authors used a finite element method such that $\Omega_{h}$ was the polygon formed by the union of all triangles of the mesh of parameter $h$. They proved an error of order $O\left(h^{3 / 2}\right)$ for the difference between continuous and discrete optimal controls. Here we show that the estimate obtained in [E. Casas and J. Sokolowski, SIAM J. Control Optim., 48 (2010), pp. 3746-3780] cannot be improved, which leads to the paradox that the numerical solution is a better approximation of the optimal control than the exact one obtained just by changing the domain from $\Omega$ to $\Omega_{h}$.
\end{abstract}

Key words. Dirichlet control, error estimates, semilinear elliptic equations, finite elements

AMS subject classifications. 49J20, 35J65

DOI. $10.1137 / 100794882$

1. Introduction. In this paper we are concerned with the approximation of the control problem

$$
\left\{\begin{array}{l}
\min J(u)=\int_{\Omega} L\left(x, y_{u}(x)\right) d x+\frac{N}{2} \int_{\Gamma} u^{2}(x) d \sigma(x) \\
\text { subject to }\left(y_{u}, u\right) \in\left(L^{\infty}(\Omega) \cap H^{1 / 2}(\Omega)\right) \times L^{\infty}(\Gamma), \\
\alpha \leq u(x) \leq \beta \text { for a.e. } x \in \Gamma,
\end{array}\right.
$$

where the state $y_{u}$ associated to the control $u$ is the solution of the Dirichlet problem

$$
\left\{\begin{aligned}
-\Delta y+a(x, y)=0 & \text { in } \Omega \\
y=u & \text { on } \Gamma .
\end{aligned}\right.
$$

$\Omega$ is an open, convex, and bounded subset of $\mathbb{R}^{2}$ with a $C^{2}$ boundary $\Gamma$. The boundedness of the control is required to deal with the nonlinearity of the state equation

*Received by the editors May 11, 2010; accepted for publication (in revised form) July 20, 2011; published electronically September 27, 2011. The first and the third authors were partially supported by the Spanish Ministry of Science and Innovation under projects MTM2008-04206 and "Ingenio Mathematica (i-MATH)" CSD2006-00032 (Consolider Ingenio 2010).

http://www.siam.org/journals/sicon/49-5/79488.html

${ }^{\dagger}$ Departmento de Matemática Aplicada y Ciencias de la Computación, E.T.S.I. Industriales y de Telecomunicación, Universidad de Cantabria, Av. Los Castros s/n, 39005 Santander, Spain (eduardo.casas@unican.es).

${ }^{\ddagger}$ Konrad-Zuse-Zentrum für Informationstechnik Berlin (ZIB), Takustraße 7, 14195 Berlin, Germany (guenther@zib.de).

$\S$ Departmento de Matemáticas, E.U.I.T.I. de Gijón, Universidad de Oviedo, Campus de Gijón, 33203 Gijón, Spain (mmateos@uniovi.es).

1998 
and the integrand $L\left(x, y_{u}\right)$, but it is not necessary for linear equations and functionals $L$ with a quadratic polynomial growth in $y$.

To solve this problem it is necessary to approximate $\Omega$ by a simpler domain (typically polygonal) $\Omega_{h}$ with a boundary $\Gamma_{h}$. In a recent paper Casas and Sokolowski [4] studied the influence of the replacement of $\Omega$ by $\Omega_{h}$ on the solutions of the control problems. To this aim, a polygonal approximation $\Omega_{h}$ of $\Omega$ was considered, $h$ being the maximum length of the sides of the polygon. Then a one-to-one mapping $g_{h}: \Gamma_{h} \longrightarrow \Gamma$ was defined and a control problem $\left(\mathrm{P}_{h}\right)$ was formulated in $\Omega_{h}$ in a similar way to $(\mathrm{P})$. The convergence of the these approximations was proved in the following sense:

1. For any sequence $\left\{\bar{u}_{h}\right\}_{h>0}$ of solutions of control problems $\left(\mathrm{P}_{h}\right)$, the sequence $\left\{\bar{u}_{h} \circ g_{h}^{-1}\right\}_{h>0} \subset L^{2}(\Gamma)$ is bounded in $H^{1 / 2}(\Gamma)$ and any weak limit $\bar{u}$ is a solution of $(\mathrm{P})$.

2. For any strict local minimum of $(\mathrm{P}), \bar{u}$, there exists a sequence $\left\{\bar{u}_{h}\right\}_{h>0}$ such that $\bar{u}_{h}$ is a local solution of $\left(\mathrm{P}_{h}\right)$ and $\left\{\bar{u}_{h} \circ g_{h}^{-1}\right\}_{h>0}$ converges weakly in $H^{1 / 2}(\Gamma)$ to $\bar{u}$.

Finally, the error $\left\|\bar{u}-\bar{u}_{h} \circ g_{h}^{-1}\right\|_{L^{2}(\Gamma)} \leq C h$ was proved for local solutions of (P) and $\left(\mathrm{P}_{h}\right)$ such that $\bar{u}_{h} \circ g_{h}^{-1} \rightarrow \bar{u}$ weakly in $H^{1 / 2}(\Gamma)$.

On the other hand, Deckelnick, Günther, and Hinze [5] studied the problem (P) without the nonlinear term in the state equation, $a(x, y)=-f(x)$, with $f \in L^{2}(\Omega)$, and taking $L(x, y)=\frac{1}{2}\left(y-y_{d}(x)\right)^{2}$ in the cost functional. Their goal was different: they discretized the control problem by using finite elements associated to a triangulation of the polygonal domain $\Omega_{h}$. In this case the control problems (P) and $\left(\mathrm{P}_{h}\right)$ have a unique solution $\bar{u}$ and $\bar{u}_{h}$, respectively. Under a nonrestrictive assumption in practice on the triangulation of $\Omega_{h}$, they proved the error estimate $\left\|\bar{u}-\bar{u}_{h} \circ g_{h}^{-1}\right\|_{L^{2}(\Gamma)} \leq C h^{3 / 2}$.

If we compare the results of [4] and [5], the difference is surprising. In [4] the problem $\left(\mathrm{P}_{h}\right)$ is the same as the problem (P) except for the change of domain $\Omega$ by $\Omega_{h}$, but there is no discretization of the control problem. In [5] the control problem $\left(\mathrm{P}_{h}\right)$ is a discrete problem where $\Omega$ has been replaced by $\Omega_{h}$ and the partial differential equation has been discretized so that the states $y_{u}$ are approximated by piecewise linear functions $y_{h}(u)$ solving the discrete variational equation. However, in the second case we get a better approximation to $\bar{u}$ than in the first case. The reader could conclude that the error estimates of [4] are not sharp and should be improved. In this paper we provide an example showing that the error estimates of [4] cannot be improved. Nevertheless, as predicted by the theory, the numerical computation on this example confirms the order $h^{3 / 2}$ for the difference among $\bar{u}$ and the solutions of the discrete problems. The goal of this paper is to show this paradox that reminds us of Babuska's paradox. Indeed, Babuska's paradox concerns the approximation of a simply supported circular plate, uniformly loaded, by a sequence of regular polygonal plates inscribed in the circle, also simply supported and uniformly loaded. It happens that the solutions for the polygons do not converge to that of the circle; see [1]. In the case we are considering in this paper the convergence holds, but it is not so good as the numerical approximation, which is also rather paradoxical.

The plan of the paper is as follows. In the next section we formulate an example of a control problem falling into the framework defined above, and we prove that the estimates obtained in [4] are optimal for this problem. In section 3 we describe the finite element approximation of the example and show the computational results, which confirm the theoretical estimates proved in [5]. Finally, in section 4 we explain why the numerical approach provides a better approximation of $\bar{u}$ than the exact solution of $\left(\mathrm{P}_{h}\right)$. 
2. The example. In what follows $\Omega$ will denote the unit ball of $\mathbb{R}^{2}$ centered at 0 ; then $\Gamma$ is the unit circumference. In this domain we consider the control problem

$$
\left\{\begin{array}{l}
\min J(u)=\int_{\Omega} 4 y_{u}(x) d x+\frac{1}{2} \int_{\Gamma} u^{2}(x) d \sigma(x) \\
\text { subject to }\left(y_{u}, u\right) \in\left(L^{2}(\Omega) \cap H^{1 / 2}(\Omega)\right) \times L^{2}(\Gamma),
\end{array}\right.
$$

where the state $y_{u}$ associated to the control $u$ is the solution of the Dirichlet problem

$$
\left\{\begin{aligned}
-\Delta y=0 & \text { in } \Omega \\
y=u & \text { on } \Gamma .
\end{aligned}\right.
$$

It is obvious that $(\mathrm{P})$ is strictly convex and has a unique solution $\bar{u}$ that can be characterized by the optimality system

$$
\begin{aligned}
& \left\{\begin{aligned}
-\Delta \bar{y} & =0 & & \text { in } \Omega, \\
\bar{y} & =\bar{u} & & \text { on } \Gamma,
\end{aligned}\right.
\end{aligned}
$$

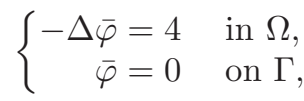

$$
\begin{aligned}
& \bar{u}=\partial_{\nu} \bar{\varphi},
\end{aligned}
$$

where $\nu(x)$ denotes the unit outward normal vector to $\Gamma$ at the point $x$. For the selected domain we have that $\nu(x)=x$. It is very easy to check that the solution of the above system is given by

$$
\bar{\varphi}(x)=1-|x|^{2}, \quad \bar{u}(x)=\partial_{\nu} \bar{\varphi}(x)=-2, \quad \text { and } \quad \bar{y}(x)=-2 .
$$

Now we define the polygonal domain $\Omega_{h}$. For every positive integer $n$ we consider the points of $\Gamma$,

$$
x_{j}=\left(\cos \frac{2 \pi j}{n}, \sin \frac{2 \pi j}{n}\right), \quad j=1,2, \ldots, n .
$$

For convenience, we set $x_{n+1}=x_{1}$. It is easy to check that the distance between two consecutive points is $h=\left|x_{j+1}-x_{j}\right|=2 \sin \frac{\pi}{n}$. We take $\Gamma_{h}$ as the polygonal line joining the knots $\left\{x_{j}\right\}_{j=1}^{n}$, and $\Omega_{h}$ is the open domain enclosed by $\Gamma_{h}$. In this domain we consider the control problem

$$
\left\{\begin{array}{l}
\min J_{h}\left(u_{h}\right)=\int_{\Omega_{h}} 4 y_{h, u_{h}}(x) d x+\frac{1}{2} \int_{\Gamma_{h}} u_{h}^{2}(x) d \sigma_{h}(x) \\
\text { subject to }\left(y_{h, u_{h}}, u_{h}\right) \in\left(L^{2}\left(\Omega_{h}\right) \cap H^{1 / 2}\left(\Omega_{h}\right)\right) \times L^{2}\left(\Gamma_{h}\right),
\end{array}\right.
$$

where the state $y_{h, u_{h}}$ associated to the control $u_{h}$ is the solution of the Dirichlet problem

$$
\left\{\begin{aligned}
-\Delta y & =0 & & \text { in } \Omega_{h} \\
y & =u_{h} & & \text { on } \Gamma_{h}
\end{aligned}\right.
$$

The previous example is inspired in another one given by Thomée [8] to prove that the estimates derived by him in the approximation of Dirichlet's problem were sharp. In fact, he considered the adjoint state equation (2.3) as the example of Dirichlet's problem. 
Problem $\left(\mathrm{P}_{h}\right)$ has a unique solution that is characterized by the system

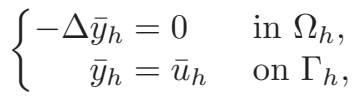

$$
\begin{aligned}
& \left\{\begin{aligned}
-\Delta \bar{\varphi}_{h}=4 & \text { in } \Omega_{h}, \\
\bar{\varphi}_{h}=0 & \text { on } \Gamma_{h},
\end{aligned}\right. \\
& \bar{u}_{h}=\partial_{\nu_{h}} \bar{\varphi}_{h},
\end{aligned}
$$

where $\nu_{h}(x)$ is the unit outward normal vector to $\Gamma_{h}$ at the point $x$; hence if $x \in$ $\left(x_{j}, x_{j+1}\right)$, then $\nu_{h}(x)=\frac{x_{j}+x_{j+1}}{\left|x_{j}+x_{j+1}\right|}$. The solution $\bar{\varphi}_{h}$ of $(2.8)$ is of class $C^{1}$ in $\bar{\Omega}_{h}$ (see section 4 for more details), and therefore $\partial_{\nu_{h}} \bar{\varphi}_{h}(x)=\nabla \bar{\varphi}_{h}(x) \cdot \nu_{h}(x)$ is well defined for every $x \in \Gamma_{h}$, with $x \neq x_{j}, j=1, \ldots, n$.

To compare the solutions $\bar{u}$ and $\bar{u}_{h}$ we introduce the mapping $g_{h}: \Gamma_{h} \longrightarrow \Gamma$ as follows. For every $1 \leq j \leq n, \widehat{x_{j} x_{j+1}}$ denotes the arc of $\Gamma$ delimited by the points $x_{j}$ and $x_{j+1}$. Then we have that $\Gamma=\cup_{j=1}^{n} \widehat{x_{j} x_{j+1}}$ and $\Gamma_{h}=\cup_{j=1}^{n}\left[x_{j}, x_{j+1}\right]$. Now we introduce a parametrization of $\widehat{x_{j} x_{j+1}}$,

$$
\psi_{j}:[0, h] \longrightarrow \widehat{x_{j} x_{j+1}} \text { is defined by } \psi_{j}(t)=x_{j}+t \tau_{j}+\phi(t) \nu_{j},
$$

where $\tau_{j}=\left(x_{j+1}-x_{j}\right) / h, \nu_{j}$ is the restriction of $\nu_{h}$ to the side $\left(x_{j}, x_{j+1}\right)$ of $\Gamma_{h}$, and $\phi:[0, h] \longrightarrow[0,+\infty)$ is chosen such that $\psi_{j}(t) \in \Gamma$. It is evident that $\phi$ is uniquely defined. Since $\Omega$ is convex and $\Gamma$ is of class $C^{2}$, the following properties hold:

1. $\phi$ is of class $C^{2}$ and $\phi(0)=\phi(h)=0$.

2. There exists a constant $C_{\Gamma}>0$ such that $\phi(t)+h\left|\phi^{\prime}(t)\right| \leq C_{\Gamma} h^{2}$ for all $t \in[0, h]$.

Finally, we define

$$
g_{h}: \Gamma_{h} \longrightarrow \Gamma,\left.\quad g_{h}\right|_{\left[x_{j}, x_{j+1}\right]}(x)=\left.g_{h}\right|_{\left[x_{j}, x_{j+1}\right]}\left(x_{j}+t \tau_{j}\right)=x_{j}+t \tau_{j}+\phi(t) \nu_{j}=\psi_{j}(t) .
$$

Clearly $g_{h}$ is one-to-one. We denote by $\tau(x)$ the unit tangent vector to $\Gamma$ at the point $x$ such that $\{\tau(x), \nu(x)\}$ is a direct reference system in $\mathbb{R}^{2}$. We can obtain the expressions for these vectors from the given parametrization. If $x$ is a point of the arc $\widehat{x_{j} x_{j+1}}$, then

$$
\tau(x)=\frac{1}{\sqrt{1+\phi^{\prime}(t)^{2}}}\left(\tau_{j}+\phi^{\prime}(t) \nu_{j}\right) \text { and } \nu(x)=\frac{1}{\sqrt{1+\phi^{\prime}(t)^{2}}}\left(\nu_{j}-\phi^{\prime}(t) \tau_{j}\right),
$$

where $x=x_{j}+t \tau_{j}+\phi(t) \nu_{j}$. From these expressions and the properties of $\phi$ we deduce that

$$
\max \left\{\left|\tau\left(g_{h}(x)\right)-\tau_{h}(x)\right|,\left|\nu\left(g_{h}(x)\right)-\nu_{h}(x)\right|\right\} \leq C_{\Gamma} h \quad \forall x \in \Gamma_{h} .
$$

Given a function $v \in L^{1}(\Gamma)$, we have

$$
\int_{\Gamma} v(x) d \sigma(x)=\sum_{j=1}^{n} \int_{0}^{h} v\left(\psi_{j}(t)\right) \sqrt{1+\phi^{\prime}(t)^{2}} d t
$$

and

$$
\int_{\Gamma_{h}} v\left(g_{h}(x)\right) d \sigma_{h}(x)=\sum_{j=1}^{n} \int_{0}^{h} v\left(g_{h}\left(x_{j}+t \tau_{j}\right)\right) d t=\sum_{j=1}^{n} \int_{0}^{h} v\left(\psi_{j}(t)\right) d t .
$$

Copyright $@$ by SIAM. Unauthorized reproduction of this article is prohibited. 
From these expressions we deduce that

$$
\int_{\Gamma_{h}}\left|v\left(g_{h}(x)\right)\right| d \sigma_{h}(x) \leq \int_{\Gamma}|v(x)| d \sigma(x) \quad \forall v \in L^{1}(\Gamma)
$$

and

$$
\begin{aligned}
& \left|\int_{\Gamma} v(x) d \sigma(x)-\int_{\Gamma_{h}} v\left(g_{h}(x)\right) d \sigma_{h}(x)\right| \leq \sum_{j=1}^{n} \int_{0}^{h}\left|v\left(\psi_{j}(t)\right)\right|\left|1-\sqrt{1+\phi^{\prime}(t)^{2}}\right| d t \\
& \quad \leq C_{\Gamma} h^{2} \sum_{j=1}^{n} \int_{0}^{h}\left|v\left(\psi_{j}(t)\right)\right| d t \leq C_{\Gamma} h^{2} \int_{\Gamma}|v(x)| d \sigma(x) \quad \forall v \in L^{1}(\Gamma) .
\end{aligned}
$$

We also have

$$
\int_{\Gamma} v(x) d \sigma(x)=\int_{\Gamma_{h}} v\left(g_{h}(x)\right)\left|D g_{h}(x) \cdot \tau_{h}(x)\right| d \sigma_{h}(x) \quad \forall v \in L^{1}(\Gamma) .
$$

The following result is an immediate consequence of [4, Theorem 9.1]

THEOREM 2.1. Let $\bar{u}$ and $\bar{u}_{h}$ denote the solutions of problems $(\mathrm{P})$ and $\left(\mathrm{P}_{h}\right)$; then there exists a constant $C>0$, independent of $h$, such that the following estimate holds:

$$
\left\|\bar{u}-\bar{u}_{h} \circ g_{h}^{-1}\right\|_{L^{2}(\Gamma)} \leq C h .
$$

Now we prove that this estimate cannot be improved. To get an underestimate for $\bar{u}-\bar{u}_{h} \circ g_{h}^{-1}$ we use (2.4) and (2.9); then

$$
\begin{aligned}
& \bar{u}(x)-\left(\bar{u}_{h} \circ g_{h}^{-1}\right)(x)=\partial_{\nu} \bar{\varphi}(x)-\left(\partial_{\nu_{h}} \bar{\varphi}_{h} \circ g_{h}^{-1}\right)(x) \\
= & \nabla \bar{\varphi}(x)\left[\nu(x)-\nu_{h}\left(g_{h}^{-1}(x)\right)\right]+\left[\nabla \bar{\varphi}(x)-\nabla \bar{\varphi}\left(g_{h}^{-1}(x)\right)\right] \nu_{h}\left(g_{h}^{-1}(x)\right) \\
& \left.+\left[\nabla \bar{\varphi}\left(g_{h}^{-1}(x)\right)-\nabla \bar{\varphi}_{h}\left(g_{h}^{-1}(x)\right)\right] \nu_{h}\left(g_{h}^{-1}(x)\right)\right] .
\end{aligned}
$$

Using that $\bar{\varphi}=0$ on $\Gamma$ and (2.10) we get that

$$
\begin{aligned}
& \left|\nabla \bar{\varphi}(x)\left[\nu(x)-\nu_{h}\left(g_{h}^{-1}(x)\right)\right]\right|=\left|\left[1-\nu(x) \cdot \nu_{h}\left(g_{h}^{-1}(x)\right)\right] \nabla \bar{\varphi}(x) \nu(x)\right| \\
= & \frac{1}{2}\left|\nu(x)-\nu_{h}\left(g_{h}^{-1}(x)\right)\right|^{2}\left|\partial_{\nu} \bar{\varphi}(x)\right|=\left|\nu(x)-\nu_{h}\left(g_{h}^{-1}(x)\right)\right|^{2} \leq C_{\Gamma}^{2} h^{2} .
\end{aligned}
$$

On the other hand, from the definition of $g_{h}$ and the properties of $\phi$ we get

$$
\begin{aligned}
\left|\left[\nabla \bar{\varphi}(x)-\nabla \bar{\varphi}\left(g_{h}^{-1}(x)\right)\right] \nu_{h}\left(g_{h}^{-1}(x)\right)\right| & =\left|-2\left[x-g_{h}^{-1}(x)\right] \nu_{h}\left(g_{h}^{-1}(x)\right)\right| \\
& =2 \phi(t)\left|\nu_{j} \cdot \nu_{h}\left(g_{h}^{-1}(x)\right)\right| \leq 2 C_{\Gamma} h^{2} .
\end{aligned}
$$

From (2.15), (2.16), and (2.17) we conclude

$$
\begin{aligned}
& \left\|\partial_{\nu_{h}} \bar{\varphi} \circ g_{h}^{-1}-\partial_{\nu_{h}} \bar{\varphi}_{h} \circ g_{h}^{-1}\right\|_{L^{2}(\Gamma)}-\left(2+C_{\Gamma}\right) C_{\Gamma} \sqrt{2 \pi} h^{2} \leq\left\|\bar{u}-\bar{u}_{h} \circ g_{h}^{-1}\right\|_{L^{2}(\Gamma)} \\
\leq & \left\|\partial_{\nu_{h}} \bar{\varphi} \circ g_{h}^{-1}-\partial_{\nu_{h}} \bar{\varphi}_{h} \circ g_{h}^{-1}\right\|_{L^{2}(\Gamma)}+\left(2+C_{\Gamma}\right) C_{\Gamma} \sqrt{2 \pi} h^{2} .
\end{aligned}
$$

Then it is enough to prove the existence of a constant $C>0$, independent of $h$, such that

$$
\left\|\partial_{\nu_{h}} \bar{\varphi} \circ g_{h}^{-1}-\partial_{\nu_{h}} \bar{\varphi}_{h} \circ g_{h}^{-1}\right\|_{L^{2}(\Gamma)} \geq C h,
$$

Copyright (c) by SIAM. Unauthorized reproduction of this article is prohibited. 
to conclude that the estimate (2.14) is sharp for the controls. But, according to (2.11), the last inequality holds if

$$
\left\|\partial_{\nu_{h}} \bar{\varphi}-\partial_{\nu_{h}} \bar{\varphi}_{h}\right\|_{L^{2}\left(\Gamma_{h}\right)} \geq C^{\prime} h
$$

for some $C^{\prime}>0$ independent of $h$.

Let us prove (2.18). To this end, we set $\phi_{h}=\bar{\varphi}-\bar{\varphi}_{h}$; then from (2.3) and (2.8) we get

$$
\left\{\begin{aligned}
-\Delta \phi_{h}=0 & \text { in } \Omega_{h} \\
\phi_{h}=\bar{\varphi} & \text { on } \Gamma_{h} .
\end{aligned}\right.
$$

Following Kenig [7, p. 121], $\phi_{h} \in H^{3 / 2}\left(\Omega_{h}\right)$ and the following estimates hold:

$$
\begin{aligned}
C_{1}\left\|\phi_{h}\right\|_{H^{1}\left(\Gamma_{h}\right)} \leq\left\|\phi_{h}\right\|_{H^{3 / 2}\left(\Omega_{h}\right)} \leq C_{1}^{\prime}\left\|\phi_{h}\right\|_{H^{1}\left(\Gamma_{h}\right)}, \\
C_{2}\left\|\partial_{\nu_{h}} \phi_{h}\right\|_{L^{2}\left(\Gamma_{h}\right)} \leq\left\|\phi_{h}\right\|_{H^{3 / 2}\left(\Omega_{h}\right)} \leq C_{2}^{\prime}\left\|\partial_{\nu_{h}} \phi_{h}\right\|_{L^{2}\left(\Gamma_{h}\right)} .
\end{aligned}
$$

From these inequalities we deduce that

$$
\left\|\partial_{\nu_{h}} \phi_{h}\right\|_{L^{2}\left(\Gamma_{h}\right)} \geq C\left\|\phi_{h}\right\|_{H^{1}\left(\Gamma_{h}\right)}=C\|\bar{\varphi}\|_{H^{1}\left(\Gamma_{h}\right)} .
$$

Hence, if we prove that $\|\bar{\varphi}\|_{H^{1}\left(\Gamma_{h}\right)} \geq C h$, then (2.18) is concluded. We have that

$$
\|\bar{\varphi}\|_{H^{1}\left(\Gamma_{h}\right)}=\left\{\|\bar{\varphi}\|_{L^{2}\left(\Gamma_{h}\right)}^{2}+\left\|\partial_{\tau_{h}} \bar{\varphi}\right\|_{L^{2}\left(\Gamma_{h}\right)}^{2}\right\}^{1 / 2} .
$$

From [2, Lemma 1] we know that the $L^{2}\left(\Gamma_{h}\right)$-norm of $\bar{\varphi}$ is of order $h^{2}$. Let us compute the norm of the tangential derivative. Since $\bar{\varphi}(x)=1-|x|^{2}$ and $\tau_{h}(x)=\frac{1}{h}\left(x_{j+1}-x_{j}\right)$ for $x \in\left(x_{j}, x_{j+1}\right)$, we have that $\partial_{\tau_{h}} \bar{\varphi}(x)=-(2 / h) x \cdot\left(x_{j+1}-x_{j}\right)$; then

$$
\begin{aligned}
& \left\|\partial_{\tau_{h}} \bar{\varphi}\right\|_{L^{2}\left(\Gamma_{h}\right)}^{2}=\sum_{j=1}^{n} \int_{x_{j}}^{x_{j+1}}\left|\frac{2}{h} x \cdot\left(x_{j+1}-x_{j}\right)\right|^{2} d \sigma_{h}(x) \\
= & \frac{4}{h} \sum_{j=1}^{n} \int_{0}^{1}\left|\left[x_{j}+t\left(x_{j+1}-x_{j}\right)\right] \cdot\left(x_{j+1}-x_{j}\right)\right|^{2} d t=\frac{4}{h} \sum_{j=1}^{n} \int_{0}^{1}\left|x_{j} \cdot x_{j+1}-1+t h^{2}\right|^{2} d t .
\end{aligned}
$$

Using that the angle between $x_{j}$ and $x_{j+1}$ is $2 \pi / n$, we get

$$
x_{j} \cdot x_{j+1}=\cos \frac{2 \pi}{n}=1-2 \sin ^{2} \frac{\pi}{n}=1-\frac{h^{2}}{2} .
$$

Therefore,

$$
\left\|\partial_{\tau_{h}} \bar{\varphi}\right\|_{L^{2}\left(\Gamma_{h}\right)}^{2}=\frac{4}{h} \sum_{j=1}^{n} \int_{0}^{1}\left|-\frac{h^{2}}{2}+t h^{2}\right|^{2} d t=4 h^{3} n \int_{0}^{1}\left(t-\frac{1}{2}\right)^{2} d t=\frac{h^{3} n}{3} .
$$

Now we observe that

$$
h=2 \sin \frac{\pi}{n} \Rightarrow h n \geq \pi \text { for } n \geq 4 .
$$

Thus, we conclude

$$
\left\|\partial_{\tau_{h}} \bar{\varphi}\right\|_{L^{2}\left(\Gamma_{h}\right)}^{2} \geq \frac{\pi h^{2}}{3}
$$

and then

$$
\|\bar{\varphi}\|_{H^{1}\left(\Gamma_{h}\right)} \geq\left\|\partial_{\tau_{h}} \bar{\varphi}\right\|_{L^{2}\left(\Gamma_{h}\right)} \geq \sqrt{\frac{\pi}{3}} h .
$$

Copyright $@$ ( ) by SIAM. Unauthorized reproduction of this article is prohibited. 
3. Finite element approximation of $(\mathbf{P})$. To get an order of convergence $O\left(h^{3 / 2}\right)$ for the finite element discretization of (P) we can use a piecewise $O\left(h^{2}\right)$ irregular family of meshes of $\Omega$ (see [5, Theorem 5.4]). To get such a family $\mathcal{T}_{h}$ we consider the regular polygon of vertices $\left(x_{j}\right)_{j=1}^{n}$ for some fixed $n \geq 4$ and the triangulation formed by the $n$ triangles of vertices $(0,0), x_{j}, x_{j+1}, j=1, \ldots, n$. Subsequent meshes are obtained by regular refinement of the initial mesh, taking into account that boundary nodes of $\Gamma_{h}$ must lie on $\Gamma$. The families obtained in this way are piecewise $O\left(h^{2}\right)$-irregular (see Remark 6.2 in [5]). In Figure 3.1 we have put the starting mesh for an octagon and two subsequent refinements of it.
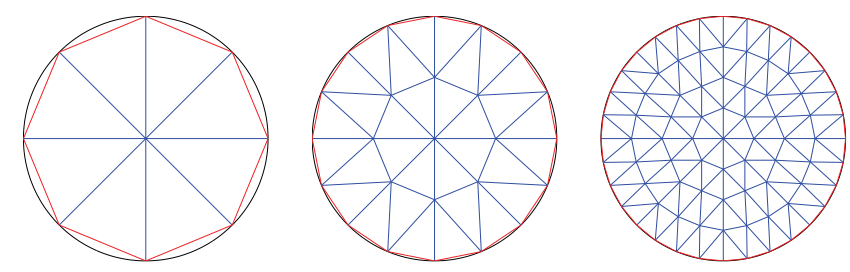

FIG. 3.1. Family of piecewise $O\left(h^{2}\right)$-irregular meshes of the circle.

Associated to $\mathcal{T}_{h}$ we consider the spaces

$$
X_{h}=\left\{\phi_{h} \in C\left(\bar{\Omega}_{h}\right): \phi_{h} \in P_{1}(T) \forall T \in \mathcal{T}_{h}\right\},
$$

$X_{h 0}=X_{h} \cap H_{0}^{1}\left(\Omega_{h}\right)$, and $U_{h}$ the restriction to $\Gamma_{h}$ of functions in $X_{h}$.

We consider the following approximation of $(\mathrm{P})$ :

$$
\left\{\begin{array}{l}
\min J_{h}\left(u_{h}\right)=\int_{\Omega_{h}} 4 y_{h}\left(u_{h}\right)(x) d x+\frac{1}{2} \int_{\Gamma_{h}} u_{h}^{2}(x) d \sigma_{h}(x) \\
\text { subject to }\left(y_{h}\left(u_{h}\right), u_{h}\right) \in X_{h 0} \times U_{h},
\end{array}\right.
$$

where the discrete state $y_{h}\left(u_{h}\right) \in X_{h}$ associated to the control $u_{h}$ is the unique solution of the following finite-dimensional problem:

$$
\text { Find } y_{h} \in X_{h} \text { s.t. } y_{h}=u_{h} \text { on } \Gamma_{h} \text { and } \int_{\Omega_{h}} \nabla y_{h} \cdot \nabla \phi_{h} d x=0 \forall \phi_{h} \in X_{h 0} \text {. }
$$

Problem $\left(\mathrm{Q}_{h}\right)$ has a unique solution $\hat{u}_{h} \in U_{h}$. Let us denote by $\hat{\varphi}_{h} \in X_{h 0}$ the discrete adjoint state associated to $\hat{u}_{h}$, characterized by the equation

$$
\int_{\Omega_{h}} \nabla \hat{\varphi}_{h} \cdot \nabla \phi_{h} d x=\int_{\Omega_{h}} 4 \phi_{h} d x \forall \phi_{h} \in X_{h 0} .
$$

Then we can write

$$
\hat{u}_{h}=\partial_{\nu_{h}}^{h} \hat{\varphi}_{h}
$$

where $\partial_{\nu_{h}}^{h} \hat{\varphi}_{h} \in U_{h}$ is the so-called discrete normal derivative, described in [3], and characterized by the system

$$
\int_{\Gamma_{h}} \partial_{\nu_{h}}^{h} \hat{\varphi}_{h} \phi_{h} d \sigma_{h}=\int_{\Omega_{h}} \nabla \hat{\varphi}_{h} \cdot \nabla \phi_{h} d x-\int_{\Omega_{h}} 4 \phi_{h} d x \forall \phi_{h} \in X_{h}
$$

Copyright (c) by SIAM. Unauthorized reproduction of this article is prohibited. 
TABLE 3.1

Mesh parameters, error, and order of convergence for full discretization. Data for meshes obtained from $i$ refinements of an octagon.

\begin{tabular}{|c|r|c|r|r|r|r|r|r|}
\hline$i$ & $n$ & $h$ & $n_{t}$ & $n_{p}$ & $E_{h}^{2}$ & $E O C^{2}$ & $E_{h}^{\infty}$ & $E O C^{\infty}$ \\
\hline 0 & 8 & 0.765367 & 8 & 9 & 0.3767136 & 0.000 & 0.152241 & 0.000 \\
1 & 16 & 0.390181 & 32 & 25 & 0.5895520 & -0.665 & 0.441659 & -1.581 \\
2 & 32 & 0.196034 & 128 & 81 & 0.2296750 & 1.370 & 0.232952 & 0.929 \\
3 & 64 & 0.098135 & 512 & 289 & 0.0881355 & 1.384 & 0.128178 & 0.863 \\
4 & 128 & 0.049082 & 2048 & 1089 & 0.0326268 & 1.434 & 0.067788 & 0.919 \\
5 & 256 & 0.024543 & 8192 & 4225 & 0.0118212 & 1.465 & 0.034916 & 0.957 \\
6 & 512 & 0.012272 & 32768 & 16641 & 0.0042341 & 1.481 & 0.017734 & 0.977 \\
7 & 1024 & 0.006136 & 131072 & 66049 & 0.0015073 & 1.490 & 0.008940 & 0.988 \\
8 & 2048 & 0.003068 & 524288 & 263169 & 0.0005349 & 1.495 & 0.004489 & 0.994 \\
9 & 4096 & 0.001534 & 2097152 & 1050625 & 0.0001895 & 1.497 & 0.002250 & 0.997 \\
\hline
\end{tabular}
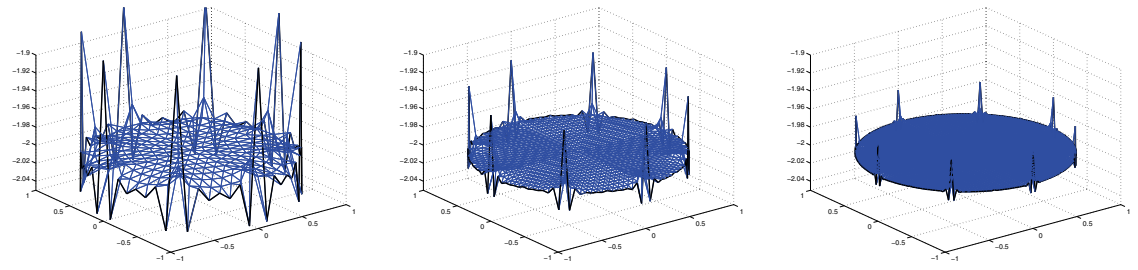

FIG. 3.2. Approximations of the discrete optimal state and the boundary control.

Since there are no control constraints, this approach is equivalent to the one given in [5], where the control is not discretized, but it is finally obtained as the pointwise projection of the discrete normal derivative.

We will take as mesh size $h$ the length of one side of $\Gamma_{h}$. For quasi-uniform meshes this is equivalent to the usual choice of the maximum edge size of the triangulation. With these settings, Theorem 5.4 in [5] states that there exists a constant $C>0$ such that

$$
\left\|\bar{u} \circ g_{h}-\hat{u}_{h}\right\|_{L^{2}\left(\Gamma_{h}\right)} \leq C h^{3 / 2} .
$$

Numerical testing confirms this order of convergence. For $p=2$ or $p=\infty$, the experimental error is given by

$$
E_{h}^{p}=\left\|\bar{u} \circ g_{h}-\hat{u}_{h}\right\|_{L^{p}\left(\Gamma_{h}\right)}
$$

and the experimental order of convergence is

$$
E O C_{h_{1}, h_{2}}^{p}=\frac{\log \left(E_{h_{1}}^{p}\right)-\log \left(E_{h_{2}}^{p}\right)}{\log \left(h_{1}\right)-\log \left(h_{2}\right)} .
$$

We obtain the results summarized in Table 3.1.

A picture of the solution for meshes obtained after successive refinements from an octagon is shown in Figure 3.2. Notice that the numerical solution has some needles located at the vertexes of the initial rough mesh. Nevertheless, these deviations are small and the convergence order in $L^{\infty}\left(\Gamma_{h}\right)$ is linear on this example.

4. Explaining the paradox. The reason for lower accuracy than expected in approximating $\bar{u}$ by $\bar{u}_{h}$ is found at the vertices $x_{j}$ of the polygonal boundary $\Gamma_{h}$. Indeed, from (2.8) we deduce that $\bar{\varphi}_{h} \in W^{2, p_{h}}\left(\Omega_{h}\right)$ for some $p_{h}>2$ depending on 
the angles of the polygonal domain $\Omega_{h}$; see Grisvard [6]. In fact, it holds for any $p_{h}<2+4 /(n-4)$, where $n$ is the number of vertices of $\Omega_{h}$. The point is that $W^{2, p_{h}}\left(\Omega_{h}\right) \subset C^{1}\left(\bar{\Omega}_{h}\right)$. Then, taking into account that $\bar{\varphi}_{h}=0$ on $\Gamma_{h}$, we have at every vertex $x_{j}$

$$
\nabla \bar{\varphi}_{h}\left(x_{j}\right) \cdot \tau_{j-1}=\nabla \bar{\varphi}_{h}\left(x_{j}\right) \cdot \tau_{j}=0,
$$

and therefore $\nabla \bar{\varphi}_{h}\left(x_{j}\right)=0$. Hence, $\nabla \bar{\varphi}_{h} \cdot \nu_{h}$ is a continuous function on $\Gamma_{h}$ if we take the value zero on the vertices $x_{j}$. Even more, we have that $\partial_{\nu_{h}} \bar{\varphi}_{h} \in W^{1-1 / p_{h}, p_{h}}\left(\Gamma_{h}\right) \subset$ $C\left(\Gamma_{h}\right)$. Therefore, $\bar{u}_{h}=\partial_{\nu_{h}} \bar{\varphi}_{h} \in W^{1-1 / p_{h}, p_{h}}\left(\Gamma_{h}\right)$ and $\bar{u}_{h}\left(x_{j}\right)=\partial_{\nu_{h}} \bar{\varphi}_{h}\left(x_{j}\right)=0$. Thus, the singularities of $\Gamma_{h}$ on the vertices $x_{j}$ force the optimal controls $\bar{u}_{h}$ to vanish on them. Taking into account that $\bar{u}\left(x_{j}\right)=-2$, we observe a big error between $\bar{u}$ and $\bar{u}_{h}$ at the vertices. Notice that the number of vertices tends to infinity when $h \rightarrow 0$ and $\left\{x_{j}\right\}_{j=1}^{n}$ becomes dense in $\Gamma$. This does not happen if we consider the numerical approximation of $\bar{u}$ on $\Gamma_{h}$. Indeed, the discrete optimal control is given by (3.1), where we use the discrete normal derivative of the discrete adjoint state; see (3.2). This discrete normal derivative does not vanish necessarily at the vertices $x_{j}$.

Let us finish by showing the solution $\bar{u}_{h}$ of $\left(\mathrm{P}_{h}\right)$. To compute $\bar{u}_{h}$ we make a finite element approximation of $\left(\mathrm{P}_{h}\right)$. For that purpose we take a quasi-uniform family of triangulations $\mathcal{T}_{\rho}^{h}$ of $\Omega_{h}$ (see Figure 4.1). Associated to $\mathcal{T}_{\rho}^{h}$ we consider the spaces

$$
X_{\rho}^{h}=\left\{\phi_{\rho} \in C\left(\bar{\Omega}_{h}\right):\left.\phi_{\rho}\right|_{T} \in P_{1}(T) \forall T \in \mathcal{T}_{\rho}^{h}\right\},
$$

$X_{\rho 0}^{h}=X_{\rho} \cap H_{0}^{1}\left(\Omega_{h}\right)$, and $U_{\rho}^{h}$ the restriction to $\Gamma_{h}$ of functions in $X_{\rho}^{h}$.
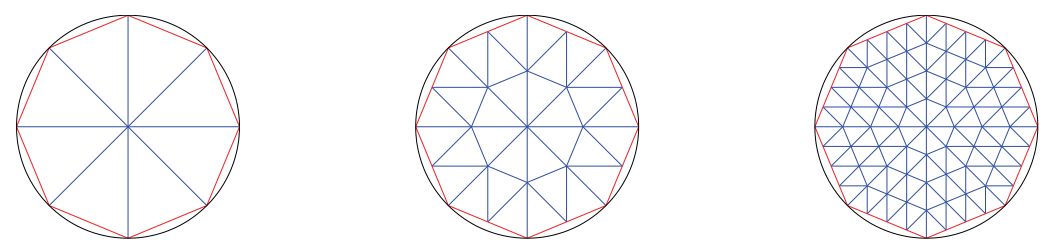

FIG. 4.1. Triangulations of the octagon for $\rho=h / 2^{k}, k=0,1,2$.

We consider the following approximation of $\left(P_{h}\right)$ :

$$
\left\{\begin{array}{l}
\min J_{\rho}^{h}\left(u_{\rho}^{h}\right)=\int_{\Omega_{h}} 4 y_{\rho}^{h}\left(u_{\rho}^{h}\right)(x) d x+\frac{1}{2} \int_{\Gamma_{h}} u_{\rho}^{h}(x)^{2} d \sigma_{h}(x) \\
\text { subject to }\left(y_{\rho}^{h}\left(u_{\rho}^{h}\right), u_{\rho}^{h}\right) \in X_{\rho 0}^{h} \times U_{\rho}^{h},
\end{array}\right.
$$

where the discrete state $y_{\rho}^{h}\left(u_{\rho}^{h}\right) \in X_{\rho}^{h}$ associated to the control $u_{\rho}^{h}$ is the unique solution of the following finite-dimensional problem:

$$
\text { Find } y_{\rho}^{h} \in X_{\rho}^{h} \text { s.t. } y_{\rho}^{h}=u_{\rho}^{h} \text { on } \Gamma_{h} \text { and } \int_{\Omega_{h}} \nabla y_{\rho}^{h} \cdot \nabla \phi_{\rho} d x=0 \forall \phi_{\rho} \in X_{\rho 0}^{h} .
$$

Problem $\left(\mathrm{Q}_{\rho}^{h}\right)$ has a unique solution $\hat{u}_{\rho}^{h} \in U_{\rho}^{h}$. Let us denote by $\hat{\varphi}_{\rho}^{h} \in X_{\rho 0}^{h}$ the discrete adjoint state associated to $\hat{u}_{\rho}^{h}$, characterized by the equation

$$
\int_{\Omega_{h}} \nabla \hat{\varphi}_{\rho}^{h} \cdot \nabla \phi_{\rho} d x=\int_{\Omega_{h}} 4 \phi_{\rho} d x \forall \phi_{\rho} \in X_{\rho 0}^{h}
$$

Copyright (C) by SIAM. Unauthorized reproduction of this article is prohibited. 

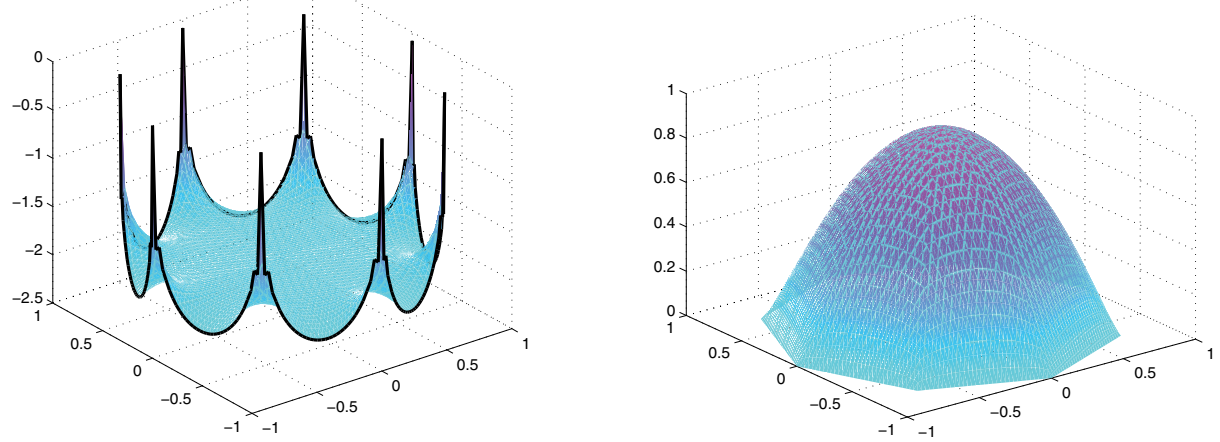

FIG. 4.2. Left: Optimal state with boundary control. Right: Associated adjoint state. $n=8$.

Then we can write

$$
\hat{u}_{\rho}^{h}=\partial_{\nu_{h}}^{\rho} \hat{\varphi}_{\rho}^{h},
$$

where $\partial_{\nu_{h}}^{\rho} \hat{\varphi}_{\rho}^{h} \in U_{\rho}^{h}$ is characterized by

$$
\int_{\Gamma_{h}} \partial_{\nu_{h}}^{\rho} \hat{\varphi}_{\rho}^{h} \phi_{\rho} d \sigma_{h}=\int_{\Omega_{h}} \nabla \hat{\varphi}_{\rho}^{h} \cdot \nabla \phi_{\rho} d x-\int_{\Omega_{h}} 4 \phi_{\rho} d x \forall \phi_{\rho} \in X_{\rho}^{h} .
$$

With these settings, Theorem 7.1 in [3] states that there exists a constant $C_{s}>0$ such that

$$
\left\|\bar{u}_{h}-\hat{u}_{\rho}^{h}\right\|_{L^{2}\left(\Gamma_{h}\right)} \leq C_{s} \rho^{s},
$$

where $s<s^{*}=\frac{1}{2}+\frac{1}{n-2}$. Figure 4.2 illustrates an approximation of the optimal solution for $n=8$.

\section{REFERENCES}

[1] I. BABUSKA, Stability of the domain under perturbation of the boundary in fundamental problems in the theory of partial differential equations principally in connection with the theory of elasticity, Parts I and II, Czechoslovak Math. J., 11 (1961), pp. 75-105, 165-203 (in Russian).

[2] J. Bramble and J. King, A robust finite element method for nonhomogeneous Dirichlet problems in domains with curved boundaries, Math. Comp., 63 (1994), pp. 1-17.

[3] E. CASAS AND J.-P. RAYMond, Error estimates for the numerical approximation of Dirichlet boundary control for semilinear elliptic equations, SIAM J. Control Optim., 45 (2006), pp. $1586-1611$.

[4] E. Casas and J. Sokolowski, Approximation of boundary control problems on curved domains, SIAM J. Control Optim., 48 (2010), pp. 3746-3780.

[5] K. Deckelnick, A. Günther, And M. Hinze, Finite element approximation of Dirichlet boundary control for elliptic PDEs on two- and three-dimensional curved domains, SIAM J. Control Optim., 48 (2009), pp. 2798-2819.

[6] P. Grisvard, Elliptic Problems in Nonsmooth Domains, Pitman, Boston, London, Melbourne, 1985.

[7] C. E. Kenig, Harmonic Analysis Techniques for Second Order Elliptic Boundary Value Problems, CBMS Regional Conf. Ser. in Math. 83, AMS, Providence, RI, 1994.

[8] V. Thomée, Polygonal domain approximation in Dirichlet's problem, J. Inst. Math. Appl., 11 (1973), pp. 33-44.

Copyright (c) by SIAM. Unauthorized reproduction of this article is prohibited. 